\title{
Utilization of Mean and Median Strains in Principal and Independent Component Analysis to Remove Motion Artefact from Electrocardiography Signals
}

\author{
Dr. Anubha Kalra ${ }^{1} \&$ Dr. Andrew Lowe ${ }^{1}$ \\ ${ }^{1}$ Institute of Biomedical Technologies, Auckland University of Technology, Auckland, NZ. \\ Correspondence: Dr. Anubha Kalra, Institute of Biomedical Technologies, Auckland University of Technology, \\ Auckland, NZ.
}

Received: April 1, 2020

doi:10.5539/mas.v14n8p1
Accepted: May 11, 2020

Online Published: May 26, 2020

URL: https://doi.org/10.5539/mas.v14n8p1

\begin{abstract}
Motion artefact is the biggest concern in Electrocardiogram signals, especially when recording long-term measurements. Current studies fail to address the major source of motion artefact, which is skin stretch. This study utilizes two-dimensional strain fields as motion information in two advanced algorithms- Principal Component Analysis (PCA) and Independent Component Analysis (ICA). The strain fields were computed using point tracking and infinitesimal strain theory and a comparison of mean and median strains as motion information was made. The highest improvement in Signal to Noise ratio (SNR) was observed when the mean values of strain fields over a region of interest per ECG sample were taken as motion information in ICA. The lowest SNRs were obtained when PCA and ICA were implemented without any motion information.
\end{abstract}

Keywords: Electrocardiogram, Principal Component Analysis, Independent Component Analysis, skin-stretch

\section{Introduction}

Electrocardiography (ECG) gives a measurement of the electrical activity of the heart over time. It is an important clinical diagnostic measure that is widely known and practiced. It can be used to diagnose conditions such as cardiac arrhythmias, murmurs, pulmonary embolism, and left ventricular hypertrophy (Bowden and Masters, 2001). In order to diagnose paroxysmal arrhythmias in ECG, it is important that it is measured over a long period of time (from several weeks to months). However, in some cases ECG is also measured on a treadmill to diagnose arrhythmia- also called stress testing. A major issue in long term measurements and stress testing is the introduction of motion artefacts which are difficult to filter out due to the same morphology and frequency as ECG. ECG with and without motion artefacts can be seen in figure 1.

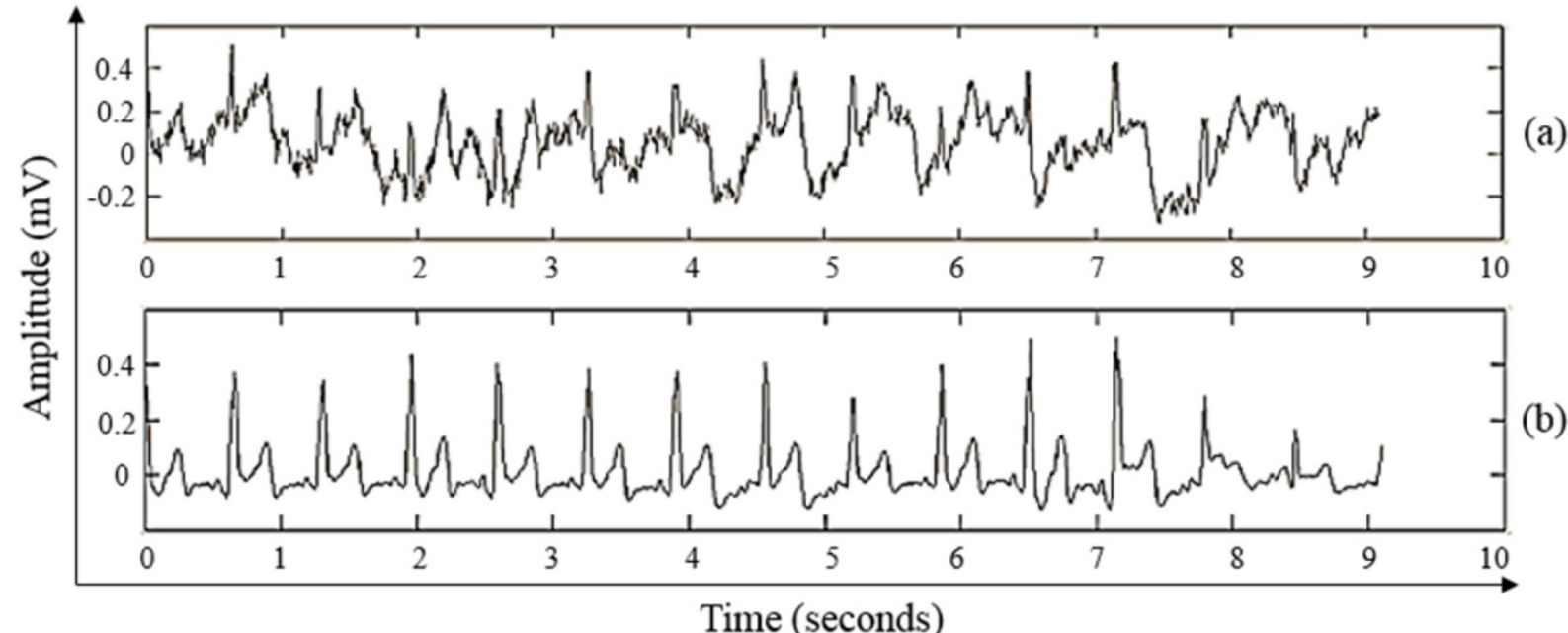

Figure 1. ECG with (a) and without (b) motion artefacts 
Existing approaches to reduce motion artefacts from ECG do not address the main cause of motion artefact, which is skin stretch. Several studies have applied algorithms to reduce motion artefact by examining only the ECG signal. Other researchers have made use of a noise signal such as uniaxial displacement, sensor acceleration or other sources which do not directly measure, or address skin stretch as a primary cause of motion artefact (Kang, 2007; Liu, 2011; Peng, 2014; Yoon et al., 2008). The optical sensing technique implemented by Liu (Liu, 2007) measured absolute displacement of the images in any direction, thereby neglecting to calculate the strain (normal and shear) of the skin.

Physical insight into strains can be obtained by decomposing them into normal and shear components. This work aims to reduce ECG motion artefacts by exploiting the physical principal of injury current due to skin strain as a primary contributor to motion artefacts. It will account for the directions and the orientation of the skin strain field at the electrode to try to achieve a high signal to noise ratio (SNR) while preserving user comfort and biocompatibility.

Finite strain theory is used to deal with arbitrarily large (finite) rigid-body displacements and rotations in elastomers, biological tissues, and fluids (Kang, 2007). This study however requires the calculation of extensional and shearing deformations; therefore, the use of infinitesimal strain theory is appropriate in this case.

This article first introduces a novel technique to quantify skin stretch using point tracking and infinitesimal strain theory and then focusses on its implementation in removing motion artefacts using advanced signal processing algorithms - Principal Component Analysis (PCA) and Independent Component Analysis (ICA). A comparison of ICA and PCA performed with and without the use of motion information is being studied.

The first part of the study involves concept validation. A Polydimethylsiloxane (PDMS) film with a checkerboard pattern was prepared and stretched using a texture analyzer. Two different strain profiles - uniform and non-uniform were introduced using rectangular and curved clamps.

The effect of PDMS stretching was quantified using infinitesimal strain theory and 2D strain analysis over different regions of interest.

The movement of the film was video recorded using a high-speed camera. For each video frame, the movement of the corner points of the checkerboard squares from the first frame was identified and tracked. The motion tracking algorithm implemented to obtain the $x-y$ coordinates of the corners of the checkerboard squares is discussed in detail in section II.

The implementation of this technique was validated with the stretch simulations carried out in SolidWorks (Systemes, 2012).

To estimate the efficiency of this proposed technique on motion artefact removal, a pilot study was also performed where a conductive polymer film made of $4 \mathrm{wt} \%$ Carbon Nano Tubes (CNT) in PDMS was prepared to measure skin stretch and ECG on a human subject. Strains in CNT/PDMS patch were evaluated and used as motion information. Motion information was incorporated in PCA and ICA to remove motion artefacts from ECG.

\section{Infinitesimal Strain Theory}

The infinitesimal strain theory is a mathematical approach in which the displacement of the material particles is smaller than any relevant dimension of the body. Estimation of the point strains can be achieved by defining a displacement field for a body deformation. In this research it is desired to estimate the strain field over a region of skin. This can be carried out by assuming that small triangular elements cover the region and estimating the strains based on the displacements of the vertices. Consider a case where some random number of test points/nodes are marked on the skin. They may be triangulated using Delaunay triangulation. Delaunay triangles are well-shaped and connect points in the nearest neighborhood and therefore Delaunay triangulation is useful in scattered data interpolation.

In order to apply this theory, it is necessary to identify points that move as the skin is stretched, so that the strain of the skin can be calculated. Therefore, the corners of the checkerboard on the PDMS were identified and tracked during the stretch and the strain fields $(\varepsilon x$, $\varepsilon y$ and $\varepsilon x y)$ at the centroids of the Delaunay triangles were calculated using equation 1. Assuming a triangular element with corners $\mathrm{ABC}$ deforms, the strain-displacement matrix can be defined using infinite strain theory as: 


$$
\left[\begin{array}{ccc}
A_{x}-B_{x} & 0 & A_{y}-B_{y} / 2 \\
0 & A_{y}-B_{y} & A_{x}-B_{x} / 2 \\
B_{x}-C_{x} & 0 & B_{y}-C_{y} / 2 \\
0 & B_{y}-C_{y} & B_{x}-C_{x} / 2 \\
C_{x}-A_{x} & 0 & C_{y}-A_{y} / 2 \\
0 & C_{y}-A_{y} & C_{x}-A_{x} / 2
\end{array}\right]\left[\begin{array}{c}
\varepsilon_{x} \\
\varepsilon_{y} \\
\varepsilon_{x y}
\end{array}\right]=\left[\begin{array}{l}
\Delta A_{x}-\Delta B_{x} \\
\Delta A_{y}-\Delta B_{y} \\
\Delta B_{x}-\Delta C_{x} \\
\Delta B_{y}-\Delta C_{y} \\
\Delta C_{x}-\Delta A_{x} \\
\Delta C_{y}-\Delta A_{y}
\end{array}\right]
$$

where $A=(A x, A y)$ and likewise $\varepsilon x, \varepsilon y \& \varepsilon x y$ are normal strain components in $\mathrm{x}, \mathrm{y}$, and shear $\mathrm{x}-\mathrm{y}$ strains respectively.

\section{Materials and Methodology}

\subsection{Concept Validation-Emulating Uniform and Non-Uniform Strain Profiles}

PDMS Sylgard 184 consisting of Part A: Base (B) and Part B: Curing Agent (CA) were mixed in ratio 10:1 in a petri dish, and the mixture was cured at $80^{\circ} \mathrm{C}$ for 2 hours. A checkerboard pattern was inked on the PDMS patch which was then subjected to tensile testing using a texture analyzer. The tensile tests were conducted at a speed of $0.5 \mathrm{~mm} / \mathrm{s}$, using rectangular and curved clamps to induce different strain fields in the membrane (Figure 2).

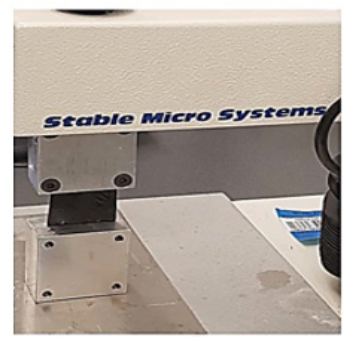

Rectangular Clamps

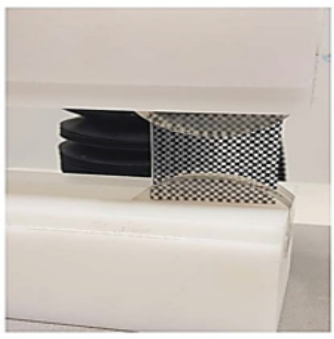

Curved Clamps

Figure 2. Tensile testing using rectangular and curved clamps

Corner points of the checkerboard were identified using the Harris-Stephens algorithm in MATLAB and their movement was tracked over consecutive frames using Euclidean distance point mapping (Kalra et al., 2015) to match points from the initial frame to the next frame by considering the minimum projected distance between them (Figure 3).

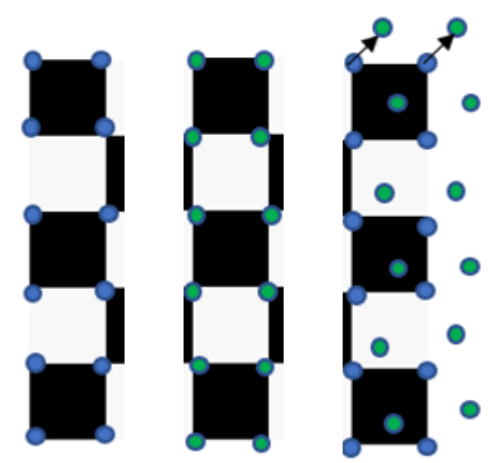

Corners in $1^{\text {st }}$ frame Corners in $2^{\text {nd }}$ frame Corners displacement Blue dots Green dots from blue to green dots

Figure 3. Euclidean distance method to track corner points on stretching

The displacements of the points from their initial positions were computed and the three components of strain $(\varepsilon x, \varepsilon y$, and $\varepsilon x y)$ were calculated using the three points on each triangle via equation 1 . The strains were 
attributed to the incentres of the triangles formed by Delaunay triangulation.

To validate the strain fields, a thin PDMS film was modeled and simulated for strain measurements in SolidWorks using similar conditions (clamps' shapes and membrane's size).

\subsection{Pilot Study-Skin Stretch Estimation and Motion Artefact Removal Using PCA and ICA}

Motion artefacts were generated by stretching and releasing the forearm skin a plastic tube, as illustrated in Figure 4. The plastic tube was used to avoid any interference in the ECG recordings due to electrical charges arising from another person. The motion noise was quantified in terms of normal and shear strains ( $\varepsilon x, \varepsilon y$, and $\varepsilon x y$ ). The motion information per frame was processed as mean and median amplitudes of strains and was incorporated in PCA and ICA.

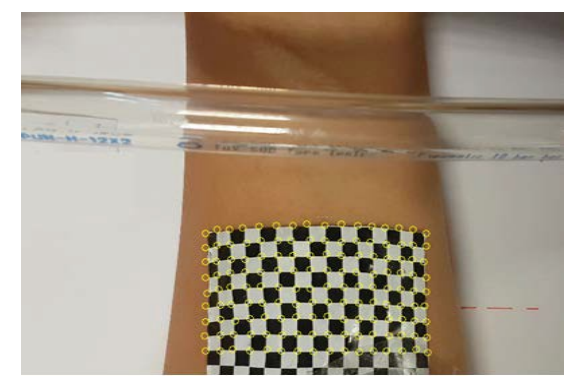

Figure 4. Strains obtained from different regions of the CNT/PDMS patch.

\section{Results}

\subsection{Comparison of Strain Fields}

The strain fields evaluated using point tracking and infinitesimal strain theory were compared with ideal simulation results. (see Figures 5 and 6). 

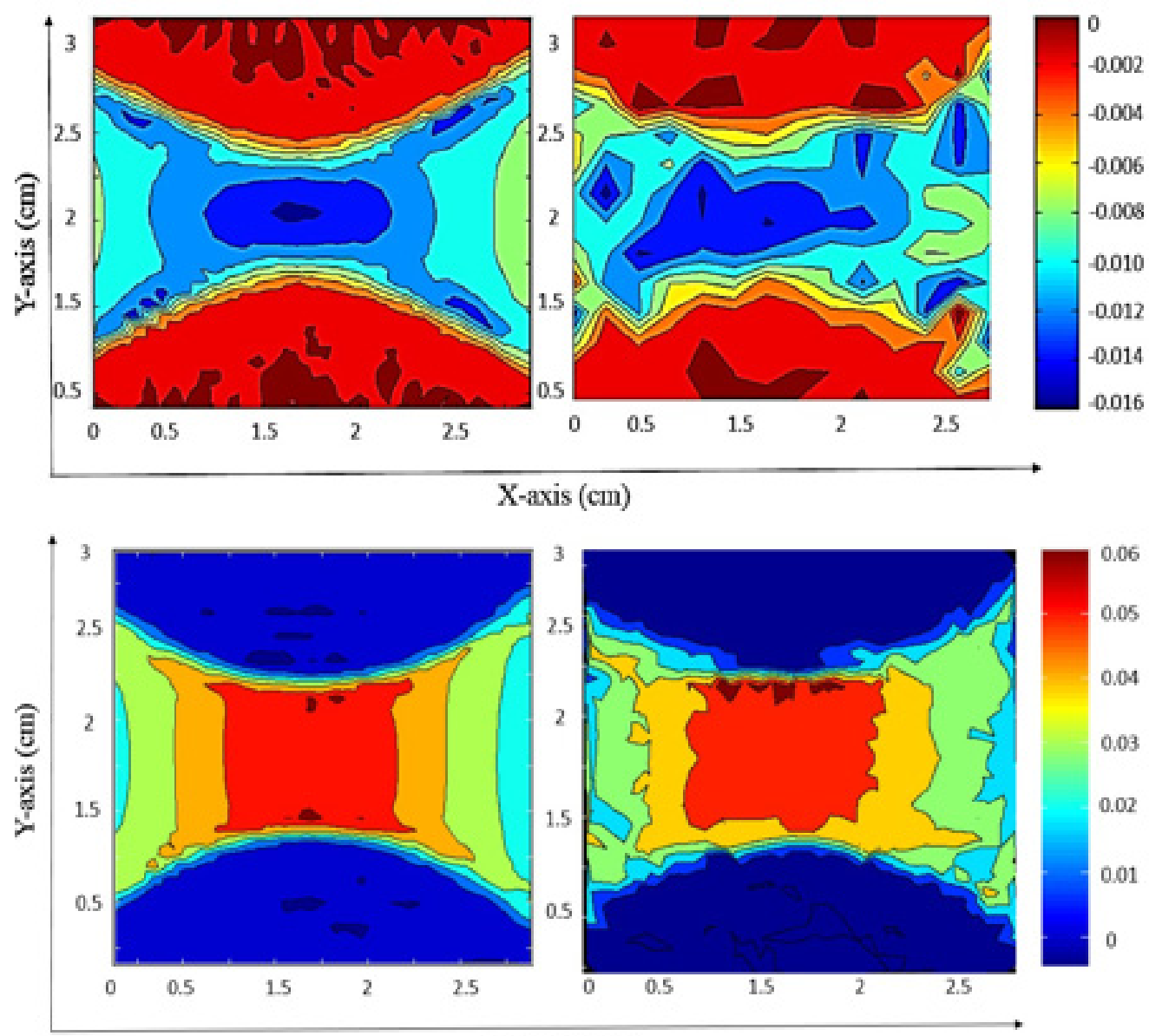

$\mathrm{X}$-axis (cm)

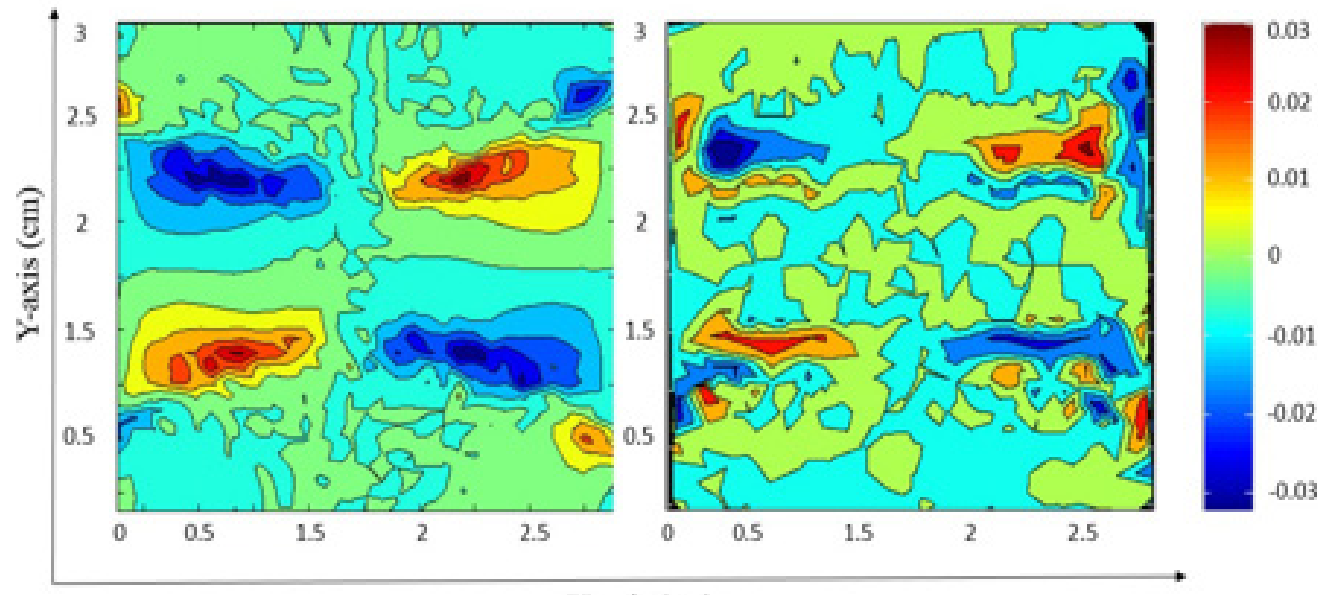

$\mathrm{X}$-axis (cm)

Figure 5. $\mathrm{X}(\varepsilon \mathrm{x}), \mathrm{Y}(\varepsilon \mathrm{gy})$, and $\mathrm{XY}(\varepsilon x y)$ plane strain distribution (from top to bottom) using circular clamps, simulated in SolidWorks (left) and estimated using point tracking technique (right). 

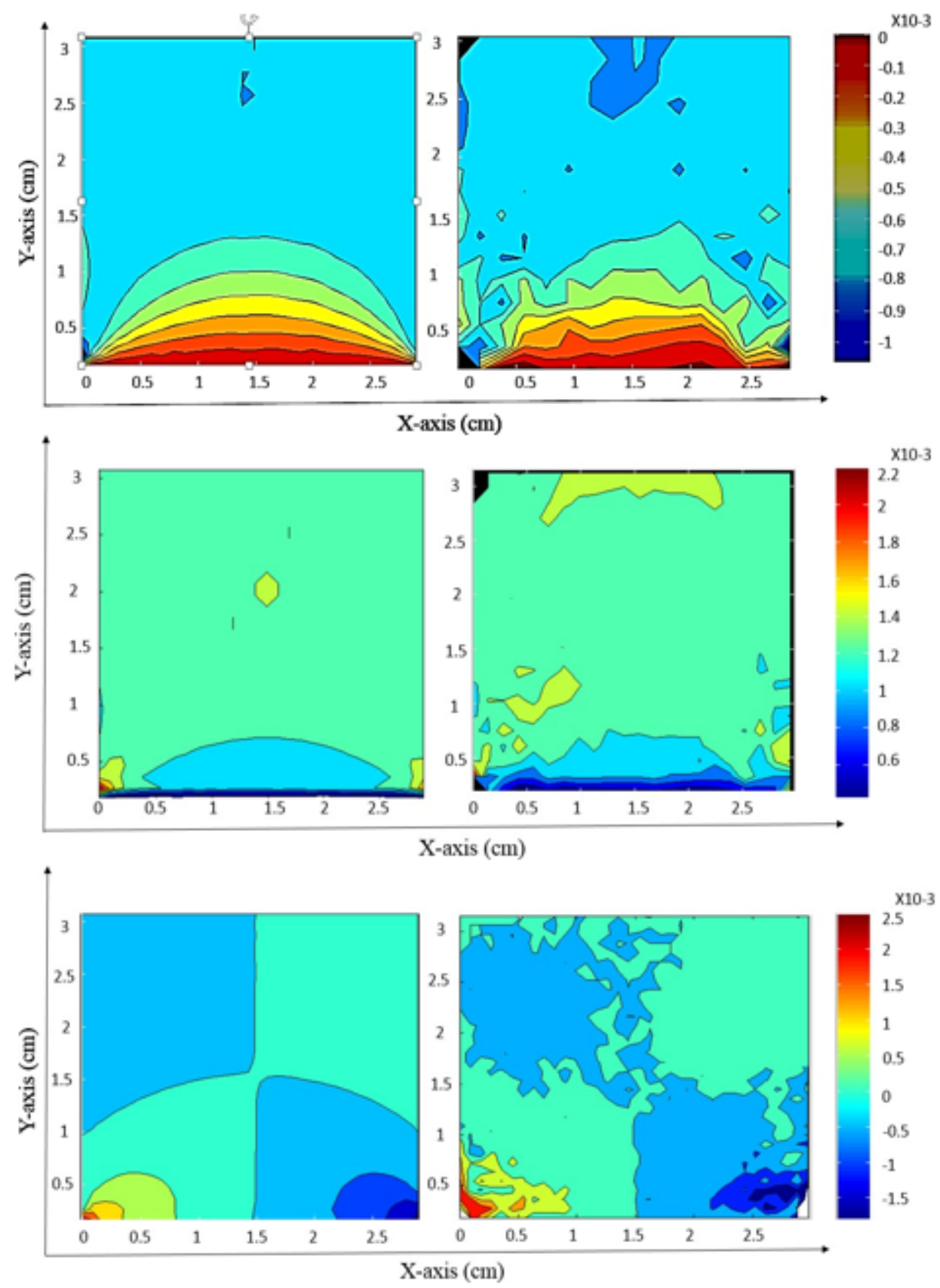

Figure 6. $\mathrm{X}(\varepsilon \mathrm{x}), \mathrm{Y}(\varepsilon \mathrm{y})$ and $\mathrm{XY}(\varepsilon x y)$ plane strain distribution (from top to bottom) using rectangular clamps, simulated in SolidWorks (left) and estimated using point tracking technique (right).

\subsection{Improvements in SNRs Using Mean and Median Strains}

A comparison between using mean and median values of strains as motion information in PCA and ICA was done to find the improvements in SNRs (Table 1).

Improvement in SNRs was calculated using the formula in equation 2. SNR noisy and SNR after PCA/ICA were calculated using equations 3 and 4 , where 'ref ECG' is the one lead ECG at rest, acquired using gel $\mathrm{Ag} / \mathrm{AgCl}$ electrodes on shoulders. 'noisy ECG' represents ECG acquired during skin-stretch, and 'ECG PCA/ICA' represents filtered ECG after using motion information in PCA and ICA. 
SNR noisy

$$
\begin{aligned}
& =10 \log _{10}\left\{\frac{\text { variance }(\text { ref } E C G)}{\text { variance }(\text { ref } E C G-\text { noisy ECG })}\right\} \\
& \text { SNR PCA ICA variance }(\text { ref ECG }) \\
& =10 \log _{10}\left\{\frac{\text { variance }(\text { ref } E C G-E C G P C A / I C A)}{\text { va }-E}\right\}
\end{aligned}
$$

Table 1. Improvements in SNRs using mean and median strains as motion information

\begin{tabular}{ccc}
\hline $\begin{array}{c}\text { Motion } \\
\text { Information in } \\
\text { the form of } \\
\text { mean/median }\end{array}$ & $\begin{array}{c}\text { SNR } \\
\text { using PCA }(d B)\end{array}$ & $\begin{array}{c}\text { SNR } \\
\text { improvement } \\
\text { using ICA }(d B)\end{array}$ \\
\hline Mean of strains & 3.25 & 10.1 \\
Median of strains & 2.75 & 6.5 \\
No motion & 1.38 & 4.417 \\
information & & \\
\hline
\end{tabular}

\section{Discussion}

Strain fields estimated using point tracking was justified and validated using simulation and experiments. The strain distribution for rectangular and circular clamps can be clearly differentiated in the above results. In the strain profiles for rectangular clamps (Figure 6) a uniform distribution of stretch induced strains can be observed for all three strain components. This is due to the equal transmission of stretch along the edge of the clamps with a monotonically increasing displacement as it is transmitted to the bottom fixture. In contrast, the strain distributions for circular clamps (Figure 5) show a radial spread throughout the film's geometry. In this case, the intensity of induced strain is centralized and decreases uniformly along the edges. The maximum strain is observed in the region with the minimum gap between the clamps and decreases as the gap increases with curvature.

The use of point strains in motion artefact removal was also implemented. The mean and median values of strains for each frame corresponding to each ECG sample were calculated to be used as motion information in PCA and ICA.

It can be observed from table 1 that both PCA and ICA performed better when provided with motion information in terms of strain fields as compared to no motion information. The performance of ICA was however better than PCA in all cases.

The mean strain amplitudes were higher as they accounted for the higher resultant displacements in certain regions unlike median strain amplitudes. It can be observed from Figure 7 that the resultant displacement of the checkerboard corners due to stretching is higher in the $\mathrm{y}$ - and xy-directions in Region B compared to that in Region A. 


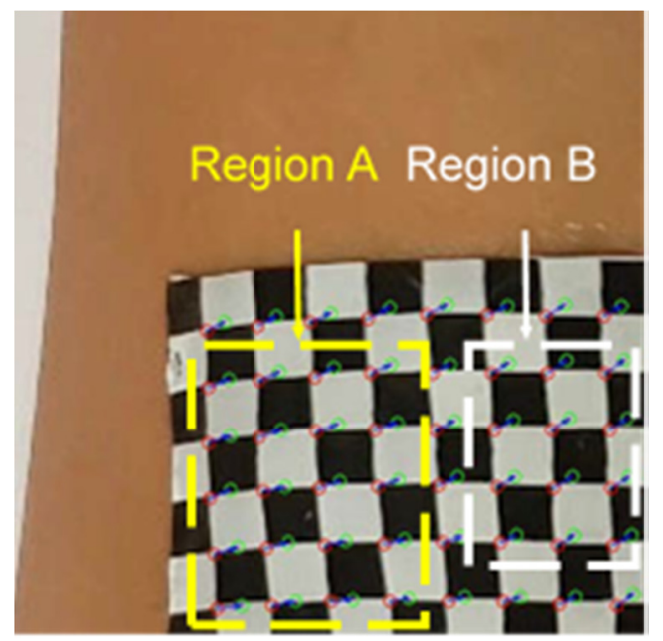

Figure 7. Displacements (blue arrows) of checkerboard corner points (in green circles) in Regions A and B from the points in the initial frame (in red circles)

\section{Conclusion}

This publication emphasizes the use of skin-stretch information for motion artefact removal from ECG. An artefact rejection system using a video camera, point tracking algorithm, infinitesimal strain theory, and signal processing algorithms-PCA and ICA have been implemented to achieve better quality ECG signals. The strain information was highly correlated with motion artefact and significantly improved the SNRs when incorporated with PCA and ICA. This technique can be used in a sensor with an in-built camera to evaluate the strain field corresponding to motion artefact. ICA showed the highest improvement in SNR (10.1dB) when mean values of strains were used as motion information corresponding to each ECG sample.

\section{Acknowledgment}

This work was fully supported by the Institute of Biomedical technologies, IBTec at Auckland University of Technology.

\section{References}

Bowden, C. \& Masters, J. (2001). Veterinary nursing medical textbook. Butterworth-Heinemann.

Kalra, A., Lowe, A. \& Al-Jumaily, A. (2015). Point Tracking on a thin elastomer to emulate Skin-stretch induced motion artifacts in Electrocardiogram measurements. 2015 International Conference on Image and Vision Computing New Zealand (IVCNZ). IEEE, 1-6. https://doi.org/10.1109/IVCNZ.2015.7761519.

Kang, T.-H. (2007). Textile-embedded sensors for wearable physiological monitoring systems.

Liu, S.-H. (2011). Motion artifact reduction in electrocardiogram using adaptive filter. J. Med. Biol. Eng, 31, 67-72. https://doi.org/10.5405/jmbe.676.

Liu, Y. (2007). Reduction of skin stretch induced motion artifacts in electrocardiogram monitoring using adaptive filtering (PhD Thesis). https://doi.org/10.1109/IEMBS.2006.260006.

Peng, G. (2014). A non-contact, low-noise electrocardiogram sensor ASIC employing motion artifact reduction.

Systemes, D. (2012). Solidworks flow simulation. Dassault Systemes. Solidworks Corporation. Velizy-Villacoublay, France.

Yoon, S.W., Min, S.D., Yun, Y.H., Lee, S. \& Lee, M. (2008). Adaptive motion artifacts reduction using 3-axis accelerometer in e-textile ECG measurement system. J. Med. Syst, 32, 101-106. https://doi.org/10.1007/s10916-007-9112-x.

\section{Copyrights}

Copyright for this article is retained by the author(s), with first publication rights granted to the journal.

This is an open-access article distributed under the terms and conditions of the Creative Commons Attribution license (http://creativecommons.org/licenses/by/3.0/). 